\title{
Optimal Motion Planning of Four-Wheeled Trailer System
}

\author{
Boyang Shi ${ }^{1}$, Haijun Peng ${ }^{1}$, Xinwei Wang ${ }^{1}$, Lingchong $\mathrm{Gao}^{2}$, Johannes Fottner ${ }^{2}$ \\ 1. Department of Engineering Mechanics, Faculty of Vehicle Engineering and Mechanics, State Key Laboratory of Structural Analysis for
} Industrial Equipment, Dalian University of Technology, Dalian, Liaoning 116024, China

E-mail: hjpeng@dlut.edu.cn

2. Chair of Materials Handling, Material Flow, Logistics, Faculty of Mechanical Engineering, Technical University of Munich, Boltzmannstrasse 15, 85748, Garching, Germany

E-mail: lingchong.gao@tum.de

\begin{abstract}
To solve the problem of optimal motion planning of four-wheeled trailer systems(FWTSs) with control constraints, a symplectic numerical method for optimal control subject to index-2 differential-algebraic equations(DAEs) with inequality constraints is developed. In the proposed method, the optimal control problem is firstly converted into a mixed nonlinear complementary problem(MNCP) with the help of variation principle and generation function theory. Then, the mixed nonlinear complementary problem is converted into equivalent nonsmooth equations. Finally, the nonsmooth equations are solved by nonsmooth Newton method. The proposed method satisfies the first-order necessary conditions and matrices involved are sparse, it is beneficial to save memory and heighten efficiency. Numerical simulations demonstrate that the proposed method is effective for motion planning of four-wheeled trailer system.
\end{abstract}

Key Words: Four-wheeled trailer system, motion planning, optimal control, differential-algebraic equations, symplectic method

\section{Introduction}

As one of the most typical class of tractor-trailer systems[1]-[3], four-wheeled trailer system(FWTS)[4]-[6] has been extensively studied for simulation of practical transport task. To enhance transport efficiency and decrease cost or fuel consumption, the motion planning of FWTSs is essential. Many algorithms have been developed to solve the motion planning of FWTSs, where optimal control methods have been paid much attention for long time because of sufficient accuracy and reasonable efficiency. Numerical methods for optimal control problems divide into two categories: direct methods and indirect methods.

In direct methods, the original continuous-time optimal control problem is discretized directly as a finite nonlinear programming(NLP)[7],[8], then the NLP can be solved by many mature algorithms. Direct methods are convenient to be programmed, since different types of constraints can be handled uniformly. However, with the increase of unknown variables, the curse of dimensionality is inevitable. In indirect methods, via variation principle and Pontryagin's maximum principle, the necessary conditions for the optimal control problem often expressed as a two-point boundary value problem(TPBVP) are derived. Thus, the optimal control problem is converted into a TPBVP. Various numerical methods such as shooting methods[7], generation function methods[9]-[11] and finite difference methods[12] are proposed. Solutions obtained by indirect methods are naturally local optimal solutions since the necessary conditions are satisfied.

From the modeling point of view, FWTS is a constrained system. Differential-algebraic equation(DAE) is an effective

*This work is supported by National Natural Science Foundation (NNSF) of China under Grant 11772074, 11761131005. tool to describe the constrained system. The formulation of DAEs is often simple and can be obtained programmatically, compared with an equivalent formulation of ordinary differential equation(ODE) which is more difficult to achieve and depends on a proper choice of coordinate. However, DAEs lead to ill-posed problems, they present numerical difficulties [13],[14] which do not occur with ODEs. Therefore, the solution and control for DAEs still confront severe challenges.

Based on the idea of indirect methods and take the advantage of description of DAEs, a symplectic numerical method for optimal control subject to DAE is proposed to deal with the motion planning of FWTSs in this paper. In the proposed method, unknown variables are approximated by Lagrange interpolation. Based on the variation principle and the fourth generation function, optimal control is converted into a mixed nonlinear complementary problem(MNCP), then it is converted into nonsmooth equations and the nonsmooth Newton method is employed to solve it.

The proposed method is applied to solving the motion planning of FWTS with control constraints successfully. Numerical simulations indicate that the proposed method is effective for optimal control subject to DAEs with control constraints. The numerical solution of state variables and algebraic variables can be obtained and control constraints are satisfied.

\section{Kinematics model of FWTS}

As depicted in Figure 1, FWTS is composed of a driven vehicle and a passive trailer, which are connected by a planar hinge. Driven by the vehicle which has two wheels powered by motor, the trailer can be transported to the specified position. 


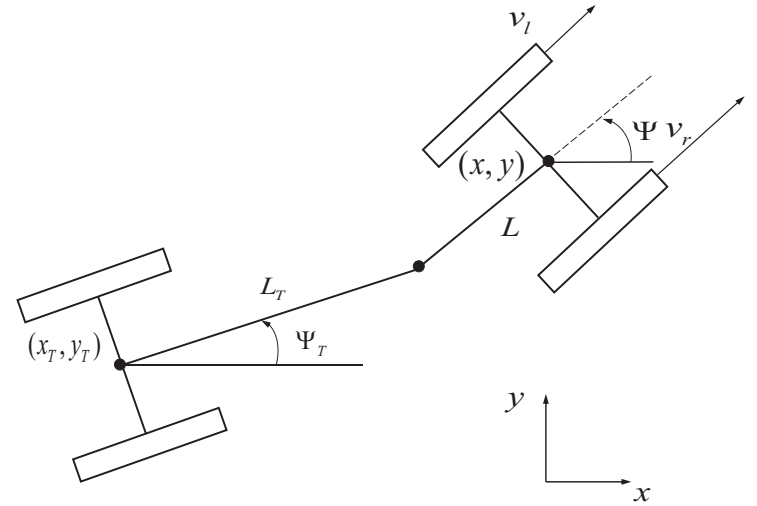

Figure 1 diagram of trailer system

Herein, the gravity center of vehicle, the gravity center of trailer, the steering angle of vehicle, the steering angle of trailer, the distance between the gravity center of vehicle and attachment point, the distance between the gravity center of trailer and attachment point, the widths of vehicle and trailer are represented as $(x, y),\left(x_{T}, y_{T}\right), \psi, \psi_{T}, L, L_{T}, B$, respectively. To describe the motion of the FWTS, the velocities of vehicle's wheels are denoted as $v_{r}$ (left) and $v_{l}$ (right) and the accelerations of vehicle's wheels which serve as control inputs are denoted as $u_{1}$ (left) and $u_{2}$ (right). Similarly, the velocities of trailer's wheels are denoted as $y_{l}$ (left) and $y_{r}$ (right), which serve as algebraic variables in following equations. Let $\boldsymbol{x}=\left[x, y, \psi, v_{l}, v_{r}, x_{T}, y_{T}, \psi_{T}\right]^{T}$ be state variables, $\boldsymbol{y}=\left[y_{l}, y_{r}\right]^{\mathrm{T}}$ algebraic variables and $\boldsymbol{u}=\left[u_{1}, u_{2}\right]^{\mathrm{T}}$ control inputs. Thus, the kinematics equations of the FWTS can be expressed as following DAE:

$$
\left\{\begin{array}{l}
\dot{\boldsymbol{x}}=\boldsymbol{f}(\boldsymbol{x}, \boldsymbol{y}, \boldsymbol{u}, t) \\
\mathbf{0}=\boldsymbol{g}(\boldsymbol{x})
\end{array}\right.
$$

where the right-hand side of differential equation is

$$
\boldsymbol{f}(\boldsymbol{x}, \boldsymbol{y}, \boldsymbol{u}, t)=\left[\begin{array}{c}
\left(v_{l}+v_{r}\right) \cos (\psi) / 2 \\
\left(v_{l}+v_{r}\right) \sin (\psi) / 2 \\
\left(v_{r}-v_{l}\right) / B \\
u_{l} \\
u_{r} \\
\left(y_{l}+y_{r}\right) \cos \left(\psi_{T}\right) / 2 \\
\left(y_{l}+y_{r}\right) \sin \left(\psi_{T}\right) / 2 \\
\left(y_{r}-y_{l}\right) / B
\end{array}\right]
$$

And the right-hand side of algebraic equation is

$$
\boldsymbol{g}(\boldsymbol{x})=\left[\begin{array}{c}
x_{T}+L_{T} \cos \left(\psi_{T}\right)-(x-L \cos (\psi)) \\
y_{T}+L_{T} \sin \left(\psi_{T}\right)-(y-L \sin (\psi))
\end{array}\right]
$$

Differentiate algebraic equation with respect to time twice, an ODE can be obtained. Thus, the differential index of Eq.(1) is two.

\section{Optimal control for FWTS}

\subsection{Problem formulation}

The motion planning of FWTS can be described as following general optimal control:
(Problem P)Find optimal control $\boldsymbol{u}^{*}$ and corresponding variables $\boldsymbol{x}^{*}$ and $\boldsymbol{y}^{*}$ to minimize the cost functional:

$$
J=\frac{1}{2} \int_{0}^{t_{f}}\|\boldsymbol{u}\|_{2}^{2} \mathrm{~d} t
$$

subject to the index-2 DAE

$$
\left\{\begin{array}{l}
\dot{\boldsymbol{x}}=\boldsymbol{f}(\boldsymbol{x}, \boldsymbol{y}, \boldsymbol{u}, t) \\
\mathbf{0}=\boldsymbol{g}(\boldsymbol{x})
\end{array}\right.
$$

the boundary conditions

$$
\boldsymbol{x}\left(t_{0}\right)=x_{0}, \boldsymbol{x}\left(t_{f}\right)=x_{f}
$$

and the control constraints

$$
\boldsymbol{h}(\boldsymbol{u}, t) \leq \mathbf{0}
$$

where $t_{f}, \boldsymbol{x}_{0}, \boldsymbol{x}_{f}$ are pre-given terminal time, initial state variable and terminal variable, respectively. Vectors $\boldsymbol{x} \in \mathbb{R}^{n_{x}}, \boldsymbol{y} \in \mathbb{R}^{n_{y}}$ and $\boldsymbol{u} \in \mathbb{R}^{n_{u}}$ denote state variables, algebraic variables and control inputs, respectively. And Functions $\boldsymbol{f}: \mathbb{R}^{n_{x}} \times \mathbb{R}^{n_{y}} \times \mathbb{R}^{n_{u}} \times \mathbb{R} \rightarrow \mathbb{R}^{n_{x}}, \boldsymbol{g}: \mathbb{R}^{n_{x}} \rightarrow \mathbb{R}^{n_{y}}$, $\boldsymbol{h}: \mathbb{R}^{n_{u}} \times \mathbb{R} \rightarrow \mathbb{R}^{n_{h}}$ are the right-hand side of differential equation, the right-hand side of algebraic equation and $n_{h}$-dimensional inequality constraints, respectively.

\subsection{Necessary conditions for optimal control}

Problem $\mathbf{P}$ is an optimal control subject to index-2 DAE with inequality constraints. For solving it, based on the idea of indirect methods for optimal control, the necessary conditions of Problem $\mathbf{P}$ need to be derived primarily.

The pre-Hamiltonian function is defined as:

$$
\begin{aligned}
\bar{H}\left(\boldsymbol{x}, \boldsymbol{y}, \boldsymbol{u}, \boldsymbol{\lambda}, \lambda_{g}, t\right):= & \|\boldsymbol{u}\|_{2}^{2}+\lambda^{\mathrm{T}} \boldsymbol{f}(\boldsymbol{x}, \boldsymbol{y}, \boldsymbol{u}, t)+ \\
& \lambda_{g}{ }^{\mathrm{T}}\left(\boldsymbol{g}_{x}^{\prime}(\boldsymbol{x}) \cdot \boldsymbol{f}(\boldsymbol{x}, \boldsymbol{y}, \boldsymbol{u}, t)\right)+\boldsymbol{\beta}^{\mathrm{T}} \boldsymbol{h}(\boldsymbol{u}, t)
\end{aligned}
$$

where $\lambda \in \mathbb{R}^{n_{x}}, \lambda_{g} \in \mathbb{R}^{n_{y}}, \boldsymbol{\beta} \in \mathbb{R}^{n_{h}}$ are costate variables, algebraic multipliers and inequality multipliers, respectively. Thus, the first-order necessary conditions of Problem $\mathbf{P}$ can be stated as:

(i)Adjoint equations

$$
\begin{aligned}
& \dot{\lambda}=-\frac{\partial \bar{H}}{\partial \boldsymbol{x}} \\
& \mathbf{0}=\frac{\partial \bar{H}}{\partial \boldsymbol{y}}
\end{aligned}
$$

(ii)Original equations

$$
\begin{gathered}
\dot{\boldsymbol{x}}=\frac{\partial \bar{H}}{\partial \lambda} \\
\mathbf{0}=\boldsymbol{g}(\boldsymbol{x})
\end{gathered}
$$

(iii)Optimality conditions

$$
\mathbf{0}=\frac{\partial \bar{H}}{\partial \boldsymbol{u}}
$$

(iv)Complementary conditions

$$
\boldsymbol{\beta}^{\mathrm{T}} \boldsymbol{h}(\boldsymbol{u}, t)=0 \quad \boldsymbol{\beta} \geq \mathbf{0} \quad \boldsymbol{h}(\boldsymbol{u}, t) \leq \mathbf{0}
$$

(v)Boundary conditions

$$
\boldsymbol{x}\left(t_{0}\right)=x_{0}, \boldsymbol{x}\left(t_{f}\right)=x_{f}
$$

The above necessary conditions consist of a TPBVP with complementary conditions from the mathematical point of view. In the sequel, a symplectic numerical method is 
developed based on necessary conditions to solve Problem P.

\section{A symplectic method for optimal control}

Assume that $\bar{H}_{u u}^{\prime \prime}$ is nonsingular, then the explicit expression of control $\boldsymbol{u}$ can be obtained by solving the optimality conditions Eq.(11):

$$
\boldsymbol{u}(t)=\boldsymbol{u}\left(\boldsymbol{x}, \boldsymbol{y}, \boldsymbol{u}, \lambda, \lambda_{g}, t\right)
$$

Substituting Eq.(14) into Eq.(8), a new Hamiltonian function $H\left(\boldsymbol{x}, \boldsymbol{y}, \boldsymbol{u}, \boldsymbol{\lambda}, \boldsymbol{\lambda}_{g}, t\right)$ which does not depend on control is obtained. Thus, Eq.(9)-Eq.(10) and Eq.(12) in necessary conditions are rewritten as:

$$
\begin{gathered}
\dot{\boldsymbol{\lambda}}=-\frac{\partial H}{\partial \boldsymbol{x}} \\
\mathbf{0}=\frac{\partial H}{\partial \boldsymbol{y}} \\
\dot{\boldsymbol{x}}=\frac{\partial H}{\partial \boldsymbol{\lambda}} \\
\mathbf{0}=\boldsymbol{g}(\boldsymbol{x}) \\
\boldsymbol{\beta}^{\mathrm{T}} \tilde{\boldsymbol{h}}\left(\boldsymbol{x}, \boldsymbol{y}, \boldsymbol{u}, \boldsymbol{\lambda}, \lambda_{g}, t\right)=0 \\
\boldsymbol{\beta} \leq \mathbf{0} \tilde{\boldsymbol{h}}\left(\boldsymbol{x}, \boldsymbol{y}, \boldsymbol{u}, \boldsymbol{\lambda}, \lambda_{g}, t\right) \leq \mathbf{0}
\end{gathered}
$$

The action within time interval $[a, b]$ is defined as:

$$
S=\int_{a}^{b} \lambda^{\mathrm{T}} \dot{\boldsymbol{x}}-H \mathrm{~d} t
$$

And the corresponding fourth generation function is defined as:

$$
V=\lambda_{a}^{\mathrm{T}} \boldsymbol{x}_{a}-\lambda_{b}^{\mathrm{T}} \boldsymbol{x}_{b}+S
$$

If the equations Eq.(15) and Eq.(17) are satisfied, the variation of Eq.(21) results in

$$
\delta V=\mathbf{x}_{a}^{\mathrm{T}} \delta \boldsymbol{\lambda}_{a}-\mathbf{x}_{b}^{\mathrm{T}} \delta \boldsymbol{\lambda}_{b}
$$

It implies that $V$ is only the function of costate variables at two ends of time interval $\lambda_{a}$ and $\lambda_{b}$. Applying the first variation formula, one has:

$$
\delta V=\left(\frac{\partial V}{\partial \lambda_{a}}\right)^{\mathrm{T}} \delta \lambda_{a}+\left(\frac{\partial V}{\partial \lambda_{b}}\right)^{\mathrm{T}} \delta \lambda_{b}
$$

Compare Eq.(23) with Eq.(22), one has:

$$
\frac{\partial V}{\partial \lambda_{a}}=\boldsymbol{x}_{a}, \frac{\partial V}{\partial \lambda_{b}}=-\boldsymbol{x}_{b}
$$

Thus, based on Eq.(16), Eq.(18), Eq.(19), Eq.(22) and Eq.(24), a symplectic method is developed to solve Problem $\mathbf{P}$ in the rest of this section.

\subsection{Discretization scheme}

To calculate the action and generation function numerically, the time discretization scheme is introduced. The whole time domain $\left[0, t_{f}\right]$ is divided into $N$ intervals equidistantly. The $j^{\text {th }}$ interval is expressed as $j^{\#}:\left[t_{j-1}, t_{j}\right]$ for $j=1,2, \ldots N$, the length of interval is $\eta=t_{f} / N$, one has $t_{N}=t_{f}$ obviously. Within the $j^{\text {th }}$ interval, two equidistant point sets are defined:

$$
\mathbb{P}_{m}=\left\{t_{j-1}=\hat{t}_{j}^{1}, \ldots, \hat{t}_{j}^{i}, \ldots, \hat{t}_{j}^{m}=t_{j}\right\}
$$

with step size $\hat{t}_{j}^{i+1}-\hat{t}_{j}^{i}=t_{f} / N(m-1), i=1,2, \ldots, m-1$.

$$
\mathbb{P}_{n}=\left\{t_{j-1}=\breve{t}_{j}^{1}, \ldots, \breve{t}_{j}^{i}, \ldots, \breve{t}_{j}^{n}=t_{j}\right\}
$$

With step size $\hat{t}_{j}^{i+1}-\hat{t}_{j}^{i}=t_{f} / N(n-1), i=1,2, \ldots, n-1$. For simplicity and convenience, the following abbreviations are used:

$$
\boldsymbol{x}\left(\hat{t}_{j}^{i}\right)=\boldsymbol{x}_{j}^{i}, \boldsymbol{y}\left(\hat{t}_{j}^{i}\right)=\boldsymbol{y}_{j}^{i}, \lambda_{g}\left(\hat{t}_{j}^{i}\right)=\lambda_{g j}^{i}, \boldsymbol{\beta}\left(\hat{t}_{j}^{i}\right)=\boldsymbol{\beta}_{j}^{i}
$$

for $i=1,2, \ldots, m$. And

$$
\begin{gathered}
\lambda\left(\breve{t}_{j}^{1}\right)=\lambda\left(t_{j-1}\right)=\lambda_{j-1}, \lambda\left(\breve{t}_{j}^{n}\right)=\lambda\left(t_{j}\right)=\lambda_{j} \\
\lambda\left(\breve{t}_{j}^{i}\right)=\lambda_{j}^{i}
\end{gathered}
$$

for $i=2,3, \ldots, n-1$.

$$
\begin{aligned}
& \boldsymbol{x}\left(t_{0}\right)=\boldsymbol{x}_{0}, \boldsymbol{x}\left(t_{N}\right)=\boldsymbol{x}_{N} \\
& \lambda\left(t_{0}\right)=\lambda_{0}, \lambda\left(t_{N}\right)=\lambda_{N}
\end{aligned}
$$

The state variables, algebraic variables, algebraic multipliers and inequality multipliers are approximated by Lagrange polynomials of degree $m-1$ that interpolate $m$ equidistant points which belong to the set $\mathbb{P}_{m}$, and costate variables are approximated by Lagrange polynomials of degree $n-1$ that interpolate $n$ equidistant points which belong to the set $\mathbb{P}_{n}$. Thus, one has:

$$
\begin{aligned}
& \boldsymbol{x}(t)=(\boldsymbol{M} \otimes \boldsymbol{I}) \overline{\boldsymbol{x}}_{j} \\
& \lambda(t)=N_{1} \boldsymbol{\lambda}_{j-1}+\left(\underline{\boldsymbol{N} \otimes \boldsymbol{I}) \overline{\boldsymbol{\lambda}}_{j}+N_{n} \lambda_{j}}\right. \\
& \boldsymbol{y}(t)=(\boldsymbol{M} \otimes \boldsymbol{I}) \overline{\boldsymbol{y}}_{j} \\
& \lambda_{g}(t)=(\boldsymbol{M} \otimes \boldsymbol{I}) \overline{\boldsymbol{\lambda}}_{g_{j}} \\
& \boldsymbol{\beta}(t)=(\boldsymbol{M} \otimes \boldsymbol{I}) \overline{\boldsymbol{\beta}}_{j}
\end{aligned}
$$

where vectors $\overline{\boldsymbol{\lambda}}_{j}, \overline{\boldsymbol{x}}_{j}, \overline{\boldsymbol{y}}_{j}, \overline{\boldsymbol{\lambda}}_{\mathrm{g}}$ and $\overline{\boldsymbol{\beta}}_{j}$ are defined by

$$
\begin{aligned}
& \overline{\boldsymbol{\lambda}}_{j}=\left\{\left(\lambda_{j}^{2}\right)^{\mathrm{T}},\left(\lambda_{j}^{3}\right)^{\mathrm{T}}, \ldots,\left(\lambda_{j}^{n-1}\right)^{\mathrm{T}}\right\}^{\mathrm{T}} \\
& \overline{\boldsymbol{x}}_{j}=\left\{\left(\boldsymbol{x}_{j}^{1}\right)^{\mathrm{T}},\left(\boldsymbol{x}_{j}^{2}\right)^{\mathrm{T}}, \ldots,\left(\boldsymbol{x}_{j}^{m}\right)^{\mathrm{T}}\right\}^{\mathrm{T}} \\
& \overline{\boldsymbol{y}}_{j}=\left\{\left(\boldsymbol{y}_{j}^{1}\right)^{\mathrm{T}},\left(\boldsymbol{y}_{j}^{2}\right)^{\mathrm{T}}, \ldots,\left(\boldsymbol{y}_{j}^{m}\right)^{\mathrm{T}}\right\}^{\mathrm{T}} \\
& \overline{\boldsymbol{\lambda}}_{g_{j}}=\left\{\left(\boldsymbol{\lambda}_{g j}^{1}\right)^{\mathrm{T}},\left(\boldsymbol{\lambda}_{g j}^{2}\right)^{\mathrm{T}}, \ldots,\left(\boldsymbol{\lambda}_{g j}^{m}\right)^{\mathrm{T}}\right\}^{\mathrm{T}} \\
& \overline{\boldsymbol{\beta}}_{j}=\left\{\left(\boldsymbol{\beta}_{j}^{1}\right)^{\mathrm{T}},\left(\boldsymbol{\beta}_{j}^{2}\right)^{\mathrm{T}}, \ldots,\left(\boldsymbol{\beta}_{j}^{m}\right)^{\mathrm{T}}\right\}^{\mathrm{T}}
\end{aligned}
$$

The symbol $\boldsymbol{I}$ denote an proper identity matrix, row vectors $\boldsymbol{M}, \underline{\boldsymbol{N}}$ and the scalars $N_{1}, N_{n}$ are defined as follows:

$$
\begin{gathered}
\boldsymbol{M}=\left[M_{1}, M_{2}, \ldots, M_{m}\right], \underline{\boldsymbol{N}}=\left[N_{2}, N_{3}, \ldots, N_{n-1}\right] \\
M_{i}(t)=\prod_{k=1, k \neq i}^{m} \frac{t-(k-1) \eta /(m-1)}{(i-k) \eta /(m-1)} \\
N_{i}(t)=\prod_{k=1, k \neq i}^{n} \frac{t-(k-1) \eta /(n-1)}{(i-k) \eta /(n-1)}
\end{gathered}
$$

The symbol " $\otimes$ " denotes the Kronecker product of two matrices. It is noted that the interpolation points of costate variables are different with those of other variables, when $m \neq n$. The costate variables $\hat{\lambda}_{j}^{i}=\lambda\left(\hat{t}_{j}^{i}\right), \hat{t}_{j}^{i} \in \mathbb{P}_{m}$ can be obtained by interpolation equation Eq.(31). 


\subsection{Symplectic approximation of necessary conditions}

By utilizing the above discretization scheme, the fourth generation function in the $j^{\text {th }}$ interval is written as:

$$
V_{j}=\lambda_{j-1}^{\mathrm{T}} \boldsymbol{x}_{j}^{1}-\lambda_{j}^{\mathrm{T}} \boldsymbol{x}_{j}^{m}+\int_{t_{j-1}}^{t_{j}} \lambda^{\mathrm{T}} \dot{\boldsymbol{x}}-H\left(\boldsymbol{x}, \boldsymbol{\lambda}, \boldsymbol{y}, \lambda_{g}, \boldsymbol{\beta}\right) \mathrm{d} t
$$

According to the above property, if Eq.(15) and Eq.(17) are satisfied in $\left[t_{j-1}, t_{j}\right]$, the fourth generation function is only the function of costate variables at two ends of time interval $\lambda_{j-1}$ and $\lambda_{j}$. Thus $\overline{\boldsymbol{x}}_{j}$ and $\bar{\lambda}_{j}$ are stationary points of $V_{j}$, stationary conditions are expressed as:

$$
\begin{aligned}
& \frac{\partial V_{j}}{\partial \overline{\boldsymbol{x}}_{j}}=\mathbf{0}, j=1,2, \ldots, N \\
& \frac{\partial V_{j}}{\partial \overline{\boldsymbol{\lambda}}_{j}}=\mathbf{0}, j=1,2, \ldots, N
\end{aligned}
$$

Applying the Eq.(24), one has:

$$
\begin{aligned}
& \frac{\partial V_{j}}{\partial \lambda_{j-1}}=\boldsymbol{x}_{j-1} \quad, j=1,2, \ldots, N \\
& \frac{\partial V_{j}}{\partial \boldsymbol{\lambda}_{j}}=-\boldsymbol{x}_{j} \quad, j=1,2, \ldots, N
\end{aligned}
$$

So far, differential equations Eq.(15) and Eq.(17) in necessary conditions are converted into Eq.(44)-(47).

For equality constraints Eq.(16) and Eq.(18), assume that in interval $\left[t_{j-1}, t_{j}\right]$ they are satisfied in point set $\mathbb{P}_{m}$, one has:

$$
\begin{array}{r}
\boldsymbol{E}_{j}=\mathbf{0} \\
\boldsymbol{G}_{j}=\mathbf{0}
\end{array}
$$

herein vector $\boldsymbol{E}_{j}=\left\{\left(\boldsymbol{e}_{j}^{1}\right)^{\mathrm{T}},\left(\boldsymbol{e}_{j}^{2}\right)^{\mathrm{T}}, \ldots,\left(\boldsymbol{e}_{j}^{m}\right)^{\mathrm{T}}\right\}^{\mathrm{T}}$ where

$$
\boldsymbol{e}_{j}^{i}=\frac{\partial H}{\partial \boldsymbol{y}}\left(\boldsymbol{x}_{j}^{i}, \boldsymbol{y}_{j}^{i}, \hat{\lambda}_{j}^{i}, \lambda_{g j}^{i}, \boldsymbol{\beta}_{j}^{i}\right)
$$

and $\boldsymbol{G}_{j}=\left\{\left(\boldsymbol{g}_{j}^{1}\right)^{\mathrm{T}},\left(\boldsymbol{g}_{j}^{2}\right)^{\mathrm{T}}, \ldots,\left(\boldsymbol{g}_{j}^{m}\right)^{\mathrm{T}}\right\}^{\mathrm{T}}$ where

$$
\boldsymbol{g}_{j}^{i}=\boldsymbol{g}\left(\boldsymbol{x}_{j}^{i}\right)
$$

For complementary conditions Eq.(19), assume that in interval $\left[t_{j-1}, t_{j}\right]$ they are satisfied in point set $\mathbb{P}_{m}$, thus one has:

$$
\left\{\begin{array}{l}
\overline{\boldsymbol{\beta}}_{j}^{\mathrm{T}} \boldsymbol{c}_{j}=0 \\
\overline{\boldsymbol{\beta}}_{j} \geq 0 \\
\boldsymbol{c}_{j} \leq 0
\end{array}, \quad j=1,2, \ldots, N\right.
$$

where inequality vector $\boldsymbol{c}_{j}=\left\{\left(\boldsymbol{h}_{j}^{1}\right)^{\mathrm{T}},\left(\boldsymbol{h}_{j}^{2}\right)^{\mathrm{T}}, \ldots,\left(\boldsymbol{h}_{j}^{m}\right)^{\mathrm{T}}\right\}^{\mathrm{T}}$ where

$$
\boldsymbol{h}_{j}^{i}=\boldsymbol{h}\left(\boldsymbol{x}_{j}^{i}, \boldsymbol{y}_{j}^{i}, \hat{\lambda}_{j}^{i}, \lambda_{g j}^{i}, \boldsymbol{\beta}_{j}^{i}, \hat{t}_{j}^{i}\right)
$$

Taking all above into account, in the $j^{\text {th }}$ interval $\left[t_{j-1}, t_{j}\right]$ the necessary conditions Eq.(15)-Eq.(19) are converted into a MNCP.

$$
\left\{\begin{aligned}
\boldsymbol{F}_{1}^{j}-\boldsymbol{x}_{j-1} & =\mathbf{0} \\
\hat{\boldsymbol{F}}^{j} & =\mathbf{0} \\
\overline{\boldsymbol{\beta}}_{j}^{\mathrm{T}} \boldsymbol{c}_{j} & =0 \quad \overline{\boldsymbol{\beta}}_{j} \geq \mathbf{0} \quad \boldsymbol{c}_{j} \leq \mathbf{0} \\
\boldsymbol{F}_{4}^{j}+\boldsymbol{x}_{j} & =\mathbf{0}
\end{aligned}\right.
$$

where $\hat{\boldsymbol{F}}^{j}=\left\{\left(\boldsymbol{F}_{2}^{j}\right)^{\mathrm{T}},\left(\boldsymbol{F}_{3}^{j}\right)^{\mathrm{T}},\left(\boldsymbol{E}_{j}\right)^{\mathrm{T}},\left(\boldsymbol{G}_{j}\right)^{\mathrm{T}}\right\}^{\mathrm{T}}, \boldsymbol{F}_{1}^{j}=\frac{\partial V_{j}}{\partial \lambda_{j-1}}$, $\boldsymbol{F}_{2}^{j}=\frac{\partial V_{j}}{\partial \overline{\boldsymbol{x}}_{j}}, \boldsymbol{F}_{3}^{j}=\frac{\partial V_{j}}{\partial \overline{\boldsymbol{\lambda}}_{j}}$ and $\boldsymbol{F}_{4}^{j}=\frac{\partial V_{j}}{\partial \lambda_{j}}$. The unknown variables in Eq.(54) are arranged as $\boldsymbol{v}_{j}=\left[\boldsymbol{\lambda}_{j-1}{ }^{\mathrm{T}}, \overline{\boldsymbol{x}}_{j}^{\mathrm{T}}, \overline{\boldsymbol{\lambda}}_{j}^{\mathrm{T}}, \overline{\boldsymbol{y}}_{j}^{\mathrm{T}}, \overline{\boldsymbol{\lambda}}_{g j}^{\mathrm{T}}, \overline{\boldsymbol{\beta}}_{j}^{\mathrm{T}}, \boldsymbol{\lambda}_{j}^{\mathrm{T}}\right]^{\mathrm{T}} \cdot$

Consider the whole time domain $\left[0, t_{f}\right]$, apply Eq.(54) for $j=1,2, \ldots, N$. The necessary conditions in $\left[0, t_{f}\right]$ are converted into a following MNCP:

$$
\left\{\begin{aligned}
\boldsymbol{F}_{1}^{1}-\boldsymbol{x}_{0} & =\mathbf{0} \\
\hat{\boldsymbol{F}}^{j} & =\mathbf{0} \\
\overline{\boldsymbol{\beta}}_{j}^{\mathrm{T}} \boldsymbol{c}_{j} & =0 \quad \overline{\boldsymbol{\beta}}_{j} \geq 0 \quad \boldsymbol{c}_{j} \leq 0 \\
\boldsymbol{F}_{4}^{l}+\boldsymbol{F}_{1}^{l+1}=\mathbf{0} & \quad l=1,2, \ldots, N-1 \\
\boldsymbol{F}_{4}^{N}+\boldsymbol{x}_{N} & =\mathbf{0}
\end{aligned}\right.
$$

with unknown variables $\boldsymbol{v}=\left[\lambda_{0}{ }^{\mathrm{T}} ; \overline{\boldsymbol{x}}_{1}^{\mathrm{T}}, \ldots, \overline{\boldsymbol{\beta}}_{1}^{\mathrm{T}}, \lambda_{1}{ }^{\mathrm{T}} ; \overline{\boldsymbol{x}}_{2}{ }^{\mathrm{T}}\right.$ $\left., \ldots, \overline{\boldsymbol{\beta}}_{2}^{\mathrm{T}}, \lambda_{2}{ }^{\mathrm{T}} ; \ldots ; \overline{\boldsymbol{x}}_{N}^{\mathrm{T}}, \ldots, \overline{\boldsymbol{\beta}}_{N}^{\mathrm{T}}, \lambda_{N}^{\mathrm{T}}\right]^{\mathrm{T}}$.

\subsection{Conversion of MNCP}

For solving MNCP derived above, based on nonsmooth theory the complementary conditions in MNCP should be converted into nonsmooth equality equations. Consider the following nonsmooth function $\varphi: \mathbb{R}^{2} \rightarrow \mathbb{R}$ defined by:

$$
\varphi(a, b)=\sqrt{a^{2}+b^{2}}-a-b
$$

it holds

$$
\varphi(a, b)=0 \quad \text { if and only if } a \geq 0, b \geq 0, a b=0
$$
Thus applying the above property Eq.(57) in the interval $\left[t_{j-1}, t_{j}\right]$, the complementary conditions in Eq.(54) are converted into $m \times n_{h}$ equations:

$$
\boldsymbol{\Phi}_{j}\left\{\left(\boldsymbol{\varphi}_{j}^{1}\right)^{\mathrm{T}},\left(\boldsymbol{\varphi}_{j}^{2}\right)^{\mathrm{T}}, \ldots,\left(\boldsymbol{\varphi}_{j}^{m}\right)^{\mathrm{T}}\right\}^{\mathrm{T}}=\mathbf{0}
$$

where $\boldsymbol{\varphi}_{j}^{k}=\left\{\left(\boldsymbol{\varphi}_{j}^{k}\right)_{1},\left(\boldsymbol{\varphi}_{j}^{k}\right)_{2}, \ldots,\left(\boldsymbol{\varphi}_{j}^{k}\right)_{n_{h}}\right\}^{\mathrm{T}} \in \mathbb{R}^{n_{h}}, k=1,2, \ldots, m$ denotes the equations corresponding to time $\hat{t}_{j}^{k}$, the $i^{\text {th }}$ component of $\varphi_{j}^{k}$ is given by:

$$
\left(\boldsymbol{\varphi}_{j}^{k}\right)_{i}=\varphi\left(-\left(\boldsymbol{h}_{j}^{k}\right)_{i},\left(\boldsymbol{\beta}_{j}^{k}\right)_{i}\right)
$$

where $\left(\boldsymbol{h}_{j}^{k}\right)_{i}$ and $\left(\boldsymbol{\beta}_{j}^{k}\right)_{i}$ denote the $i^{\text {th }}$ component of $\boldsymbol{h}_{j}^{k}$ and $\boldsymbol{\beta}_{j}^{k}$. Thus, the MNCP Eq.(54) can be converted into equations:

$$
\mathcal{F}_{j}\left(v_{j}\right)=\mathbf{0}
$$


where the right-hand side $\mathcal{F}_{j}\left(\boldsymbol{v}_{j}\right)=\left[\left(\boldsymbol{F}_{1}^{j}-\boldsymbol{x}_{j-1}\right)^{\mathrm{T}},\left(\hat{\boldsymbol{F}}^{j}\right)^{\mathrm{T}},\left(\boldsymbol{\Phi}_{j}\right)^{\mathrm{T}},\left(\boldsymbol{F}_{4}^{j}+\boldsymbol{x}_{j}\right)^{\mathrm{T}}\right]^{\mathrm{T}}$. is

Furthermore, consider the whole time interval $\left[0, t_{f}\right]$, the total MNCP Eq.(55) can be converted into the equations:

$$
\mathcal{F}(v)=0
$$

where the right-hand side is

$$
\mathcal{F}(\boldsymbol{v})=\left\{\begin{array}{c}
\left\{\left(\boldsymbol{F}_{1}^{1}-\boldsymbol{x}_{0}\right)^{\mathrm{T}},\left(\hat{\boldsymbol{F}}^{1}\right)^{\mathrm{T}},\left(\boldsymbol{\Phi}_{1}\right)^{\mathrm{T}}\right\} \\
\left\{\left(\boldsymbol{F}_{4}^{1}+\boldsymbol{F}_{1}^{2}\right)^{\mathrm{T}},\left(\hat{\boldsymbol{F}}^{2}\right)^{\mathrm{T}},\left(\boldsymbol{\Phi}_{2}\right)^{\mathrm{T}}\right\} \\
\vdots \\
\left\{\left(\boldsymbol{F}_{4}^{N-1}+\boldsymbol{F}_{1}^{N}\right)^{\mathrm{T}},\left(\hat{\boldsymbol{F}}^{N}\right)^{\mathrm{T}},\left(\boldsymbol{\Phi}_{N}\right)^{\mathrm{T}}\right\} \\
\left(\boldsymbol{F}_{4}^{N}+\boldsymbol{x}_{N}\right)^{\mathrm{T}}
\end{array}\right\}
$$

Since the component $\boldsymbol{\Phi}_{j}$ is nonsmooth, the total equations $\mathcal{F}(\boldsymbol{v})=\mathbf{0}$ are nonsmooth and a corresponding nonsmooth method needs to be adopted to solve it.

\subsection{Solution of nonsmooth equations}

The solution of optimal control can be obtained by solving Eq.(61). Since the equations are nonsmooth, the nonsmooth Newton method[15] is adopted to solve Eq.(61) instead of classical Newton method. In this method, a substitution matrix needs to be obtained to replace non-existing Jacobian matrix $\mathcal{F}^{\prime}(v)$.

For the equations $\mathcal{F}_{j}\left(\boldsymbol{v}_{j}\right)=\mathbf{0}$ within interval $\left[t_{j-1}, t_{j}\right]$, the substitution matrix can be written as:

$$
\mathcal{J}_{j}\left(\boldsymbol{v}_{j}\right)=\left[\begin{array}{c}
\frac{\partial \boldsymbol{F}_{1}^{j}}{\partial \boldsymbol{v}_{j}} \\
\frac{\partial \hat{\boldsymbol{F}}^{j}}{\partial \boldsymbol{v}_{j}} \\
\boldsymbol{L}^{j} \\
\frac{\partial \boldsymbol{F}_{4}^{j}}{\partial \boldsymbol{v}_{j}}
\end{array}\right]=\left[\begin{array}{cccc}
\boldsymbol{K}_{11}^{j} & \boldsymbol{K}_{12}^{j} & \boldsymbol{K}_{13}^{j} & \boldsymbol{K}_{14}^{j} \\
\boldsymbol{K}_{21}^{j} & \boldsymbol{K}_{22}^{j} & \boldsymbol{K}_{23}^{j} & \boldsymbol{K}_{24}^{j} \\
\boldsymbol{L}_{1}^{j} & \boldsymbol{L}_{2}^{j} & \boldsymbol{L}_{3}^{j} & \boldsymbol{L}_{4}^{j} \\
\boldsymbol{K}_{31}^{j} & \boldsymbol{K}_{32}^{j} & \boldsymbol{K}_{33}^{j} & \boldsymbol{K}_{34}^{j}
\end{array}\right]
$$

where $\boldsymbol{L}^{j}$ corresponds to the nonsmooth component $\boldsymbol{\Phi}_{j}$.

The sub matrixes of $\boldsymbol{L}^{j}$ are given by:

$$
\boldsymbol{L}_{p}^{j}=\left[\boldsymbol{L}_{1 p}^{j \mathrm{~T}}, \boldsymbol{L}_{2 p}^{j \mathrm{~T}}, \ldots, \boldsymbol{L}_{m p}^{j \mathrm{~T}}\right]^{\mathrm{T}} \quad p=1,2, . .4
$$

where

$$
\begin{gathered}
\boldsymbol{L}_{k 1}^{j}=N_{1}^{k} \boldsymbol{S}_{j}^{k} \cdot \frac{\partial \boldsymbol{h}_{j}^{k}}{\partial \hat{\lambda}_{j}^{k}} \quad k=1,2, \ldots, m \\
\boldsymbol{L}_{k 2}^{j}=\boldsymbol{S}_{j}^{k}\left[\frac{\partial \boldsymbol{h}_{j}^{k}}{\partial \boldsymbol{x}_{j}^{k}} \frac{\partial \boldsymbol{x}_{j}^{k}}{\partial \overline{\boldsymbol{x}}_{j}}, \frac{\partial \boldsymbol{h}_{j}^{k}}{\partial \hat{\boldsymbol{\lambda}}_{j}^{k}} \frac{\partial \hat{\boldsymbol{\lambda}}_{j}^{k}}{\partial \overline{\boldsymbol{\lambda}}_{j}}, \frac{\partial \boldsymbol{h}_{j}^{k}}{\partial \boldsymbol{y}_{j}^{k}} \frac{\partial \boldsymbol{y}_{j}^{k}}{\partial \overline{\boldsymbol{y}}_{j}}, \frac{\partial \boldsymbol{h}_{j}^{k}}{\partial \boldsymbol{\lambda}_{g j}{ }^{k}} \frac{\partial \boldsymbol{\lambda}_{g j}{ }^{k}}{\partial \overline{\boldsymbol{\lambda}}_{g}}\right] \\
\boldsymbol{L}_{k 3}^{j}=\left[\boldsymbol{S}_{j}^{k} \cdot \frac{\partial \boldsymbol{h}_{j}^{k}}{\partial \boldsymbol{\beta}_{j}^{k}}+\boldsymbol{R}_{j}^{k}\right) \frac{\partial \boldsymbol{\beta}_{j}^{k}}{\partial \overline{\boldsymbol{\beta}}_{j}} \quad k=1,2, \ldots, m \\
\boldsymbol{L}_{k 4}^{j}=N_{n}^{k} \boldsymbol{S}_{j}^{k} \cdot \frac{\partial \boldsymbol{h}_{j}^{k}}{\partial \hat{\boldsymbol{\lambda}}_{j}^{k}} \quad k=1,2, \ldots, m
\end{gathered}
$$

where $\boldsymbol{S}_{j}^{k}=\operatorname{diag}\left(s_{1}, s_{2}, \ldots, s_{n_{h}}\right), \boldsymbol{R}_{j}^{k}=\operatorname{diag}\left(r_{1}, r_{2}, \ldots, r_{n_{h}}\right)$. The diagonal elements are obtained by:

$$
\begin{aligned}
& s_{i}= \begin{cases}1 & \text { if }\left(\boldsymbol{\beta}_{j}^{k}\right)_{i}=0,\left(\boldsymbol{h}_{j}^{k}\right)_{i}=0 \\
\frac{\left(\boldsymbol{h}_{j}^{k}\right)_{i}}{\sqrt{\left(\boldsymbol{\beta}_{j}^{k}\right)_{i}^{2}+\left(\boldsymbol{h}_{j}^{k}\right)_{i}^{2}}}+1 & \text { otherwise }\end{cases} \\
& r_{i}= \begin{cases}0 & \text { if }\left(\boldsymbol{\beta}_{j}^{k}\right)_{i}=0,\left(\boldsymbol{h}_{j}^{k}\right)_{i}=0 \\
\frac{\left(\boldsymbol{\beta}_{j}^{k}\right)_{i}}{\sqrt{\left(\boldsymbol{\beta}_{j}^{k}\right)_{i}^{2}+\left(\boldsymbol{h}_{j}^{k}\right)_{i}^{2}}}-1 & \text { otherwise }\end{cases}
\end{aligned}
$$

Except for $\boldsymbol{\Phi}_{j}$, the components $\boldsymbol{F}_{1}^{j}, \hat{\boldsymbol{F}}^{j}, \boldsymbol{F}_{4}^{j}$ are smooth with respect to unknown variables. Thus, the corresponding components $\frac{\partial \boldsymbol{F}_{1}^{j}}{\partial \boldsymbol{v}_{j}}, \frac{\partial \hat{\boldsymbol{F}}^{j}}{\partial \boldsymbol{v}_{j}}$ and $\frac{\partial \boldsymbol{F}_{4}^{j}}{\partial \boldsymbol{v}_{j}}$ can be obtained by derivation operation.

Consider the total equations $\mathcal{F}(\boldsymbol{v})=\mathbf{0}$, applying the Eq.(63) for $j=1,2, \ldots, N$. The global substitution matrix $\mathcal{J}(v)$ is obtained

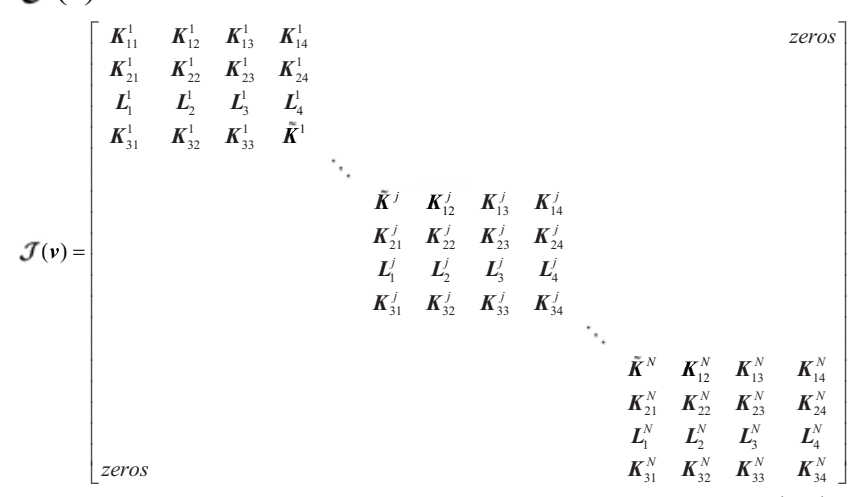

where $\tilde{\boldsymbol{K}}^{j}=\boldsymbol{K}_{34}^{j-1}+\boldsymbol{K}_{11}^{j}$ for $j=1, \ldots, N$. The substitution matrix $\mathcal{J}(v)$ which is sparse belongs to the generalized Jacobian of $\mathcal{F}(v)$ expressed as $\partial^{*} \mathcal{F}(\boldsymbol{v})$, which secure the convergence of following algorithm. Thus, the nonsmooth Newton method for Eq.(61) is described as:

\section{Algorithm 1.}

Input: Given initial solution $\boldsymbol{v}^{(0)}$, tolerance $\varepsilon$, the number of interval $N$ and interpolation parameters $m, n$

for $k=0,1,2, \ldots$ do

if $\left\|\mathcal{F}\left(v^{(k)}\right)\right\|_{2} \leq \varepsilon, v^{(k)} \rightarrow \boldsymbol{v}^{*}$ stop

else modify $\mathcal{J}\left(\boldsymbol{v}^{(k)}\right)$ and $\mathcal{F}\left(\boldsymbol{v}^{(k)}\right)$ according to (6)

calculate search direction $\boldsymbol{d}^{(k)}=-\mathcal{J}\left(\boldsymbol{v}^{(k)}\right)^{-1} \mathcal{F}\left(\boldsymbol{v}^{(k)}\right)$

and step size in search $\alpha^{(k)}$ using a proper method.

update solution $\boldsymbol{v}^{(k+1)}=\boldsymbol{v}^{(k+1)}+\alpha^{(k)} \boldsymbol{d}^{(k)}$

Output: solution $v^{*}$

Using the Algorithm 1, numerical solutions containing $\boldsymbol{x}, \boldsymbol{y}, \lambda_{,} \lambda_{g}, \boldsymbol{\beta}$ are obtained. Finally, utilizing Eq.(14), numerical solution of control $\boldsymbol{u}$ is obtained and Problem $\mathbf{P}$ is solved. 


\section{Numerical simulation}

In the numerical simulations of FWTS, according to physical limit in practical situation, the following control constraints are considered:

$$
u_{1}^{2}-u_{\max }^{2} \leq 0, u_{2}^{2}-u_{\max }^{2} \leq 0
$$

The following model parameters are used in simulation: Table 1 Model parameters

\begin{tabular}{|c|c|c|}
\hline \hline Symbol & Value & Unit \\
\hline$L$ & 0.1 & $\mathrm{~m}$ \\
\hline$L_{\mathrm{T}}$ & 0.2 & $\mathrm{~m}$ \\
\hline$B$ & 0.11 & $\mathrm{~m}$ \\
\hline$u_{\max }$ & 2.2 & $\mathrm{~N}$ \\
\hline$t_{f}$ & 2.5 & $\mathrm{~s}$ \\
\hline
\end{tabular}

The aim is to transport FWTS from a given initial position to a given terminal position and corresponding boundary conditions are given by:

$$
\begin{aligned}
& \boldsymbol{x}_{0}=[0,0,0,0,0,-0.3,0,0]^{\mathrm{T}} \\
& \boldsymbol{x}_{f}=[1,2,0,0,0,0.7,2,0]^{\mathrm{T}}
\end{aligned}
$$

For solving motion planning of FWTS, in our proposed method the algorithm parameters are chosen as $N=20, m=7, n=m+1$ and $\varepsilon=1 \times 10^{-4}$. To test the validity and efficiency of proposed method, DYNOPT 4.3 as a reference method is also used to solve the motion planning of FWTS. Correspondingly, in DYNOPT 4.3[16] the number of intervals is chosen as $N_{r}=20$ and both the number of state variables and the number of control inputs in each interval are chosen as $n x r=n u r=7$. The convergence tolerance is set as $1 \times 10^{-4}$.

The simulation is performed in MATLAB(R2014a) on an Intel ${ }^{\circledR}$ Core $^{\mathrm{TM}}$ i7-6700 machine with a $3.40 \mathrm{GHz}$ processor and $8 \mathrm{~GB}$ of RAM. In proposed method, converged solutions are obtained after 59 iterations which takes $616.2 \mathrm{~s}$ and cost functional is 6.2959. In DYNOPT 4.3, converged solutions are obtained after 845 iterations which takes $8435.9 \mathrm{~s}$ and cost functional is 6.2916. It indicates that the computational cost of proposed method is lower.

For comparison, the results of state variables and algebraic variables obtained by two methods are plotted in Figure 2, where quantities with superscript " $r$ " denotes results obtained by DYNOPT 4.3. From Figure 2(a) and (e), it can be seen that the boundary conditions Eq.(73) and Eq.(74) are satisfied accurately which indicates the FWTS arrives and stops at specified terminal position. Results obtained by proposed method has a good agreement with those obtained by DYNOPT 4.3.

Furthermore, control inputs obtained by two methods are plotted in Figure 3. Similarly, quantities with superscript "r" denotes results obtained by DYNOPT 4.3. The component $u_{1}$ increases with respect to time until $0.4 \mathrm{~s}$ and it equals maximum $0.2 \mathrm{~N}$ from $0.4 \mathrm{~s}$ to $0.8 \mathrm{~s}$. Then it decreases with respect to time and reaches minimum $-2.2 \mathrm{~N}$ at about $1.75 \mathrm{~s}$. It keeps minimum from $1.75 \mathrm{~s}$ to $2.4 \mathrm{~s}$. Finally, it increases again and reaches about $-0.21 \mathrm{~N}$ at time $2.5 \mathrm{~s}$. Components $u_{2}$ keeps maximum $2.2 \mathrm{~N}$ until $0.5 \mathrm{~s}$. Then it decreases with respect to time from $0.5 \mathrm{~s}$ to $1.7 \mathrm{~s}$ and increases modestly from $1.7 \mathrm{~s}$ to $2.1 \mathrm{~s}$. Finally, it decreases to minimum $-2.2 \mathrm{~N}$ at
$2.28 \mathrm{~s}$ and keeps it until 2.5s. Control inputs obtained by proposed method are almost consistent with those obtained by DYNOPT 4.3. However, control inputs obtained by DYNOPT 4.3 exceed control constraints slightly in some time intervals and there are some points of discontinuity on control curves. The control inputs obtained by proposed method satisfy control constraints strictly and no obvious points of discontinuity can be found.
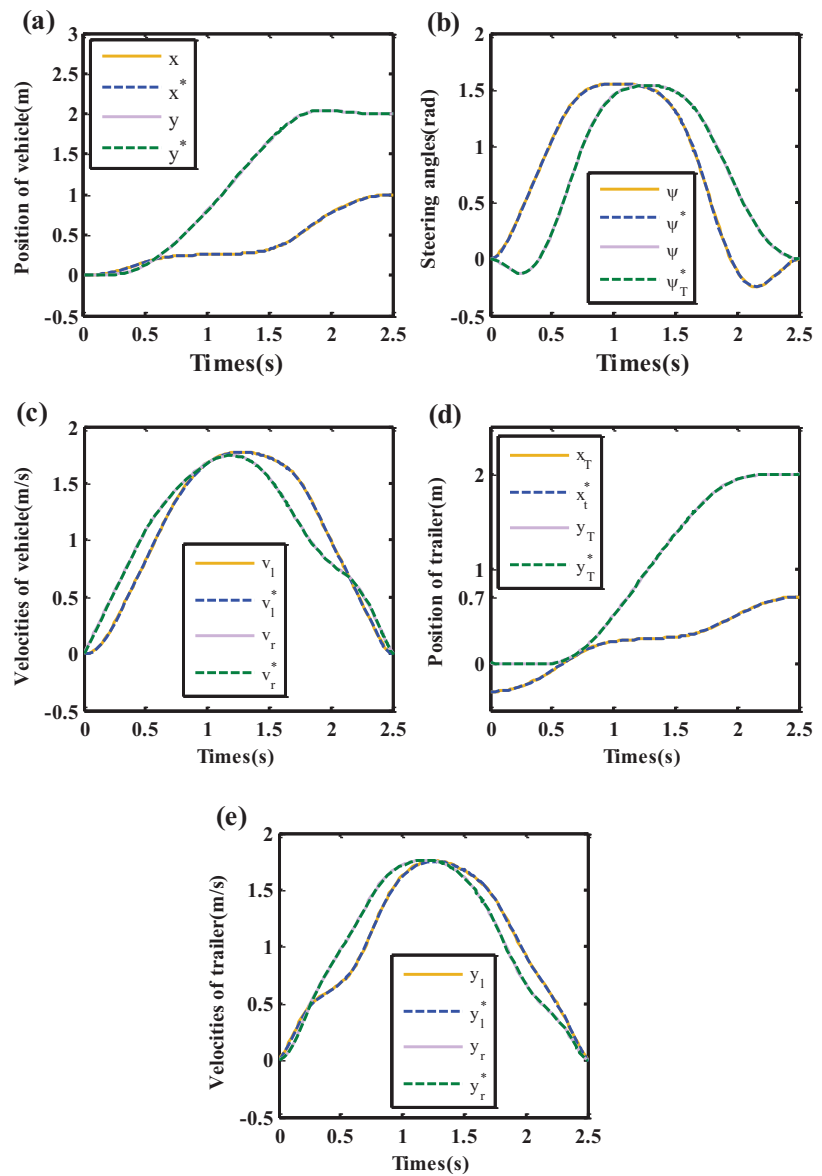

Figure 2 Results of motion planning for FWTS, including (a) position of vehicle, (b) yaw angles of vehicle and trailer, (c) velocities of vehicle, (d) position of trailer and (e) velocities of trailer(algebraic variables)

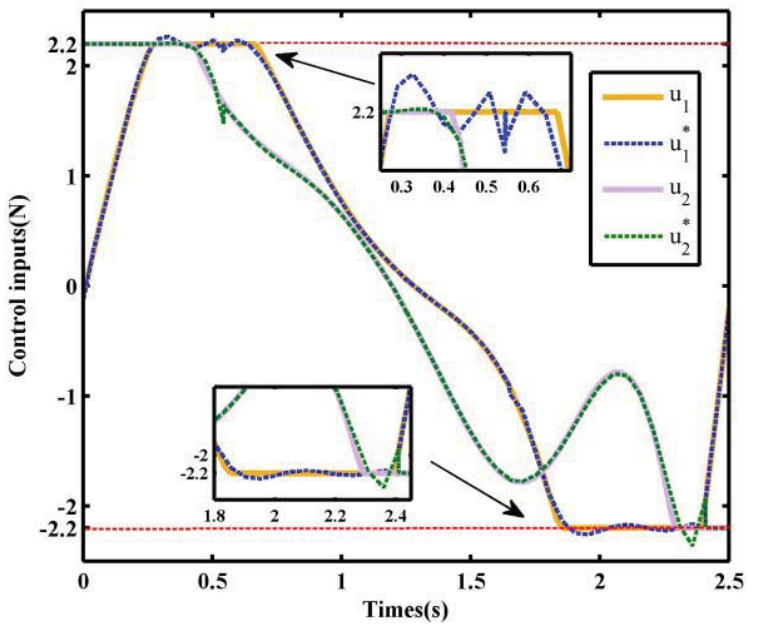

Figure 3 Control inputs of FWTS

From above analysis, the proposed method can produce high precision numerical solutions for motion planning of 
FWTS with reasonable computation cost. To present the motion trajectory of FWTS visually, the trajectory of trailer and system posture at time $t=0 \mathrm{~s}, t=1 \mathrm{~s}, t=1.25 \mathrm{~s}, t=1.5 \mathrm{~s}$ and $t=2.5 \mathrm{~s}$ are plotted in Figure 4, where blue line, green line and red line represent the trajectories of left wheel of trailer, gravity center of trailer and right wheel of trailer, respectively.

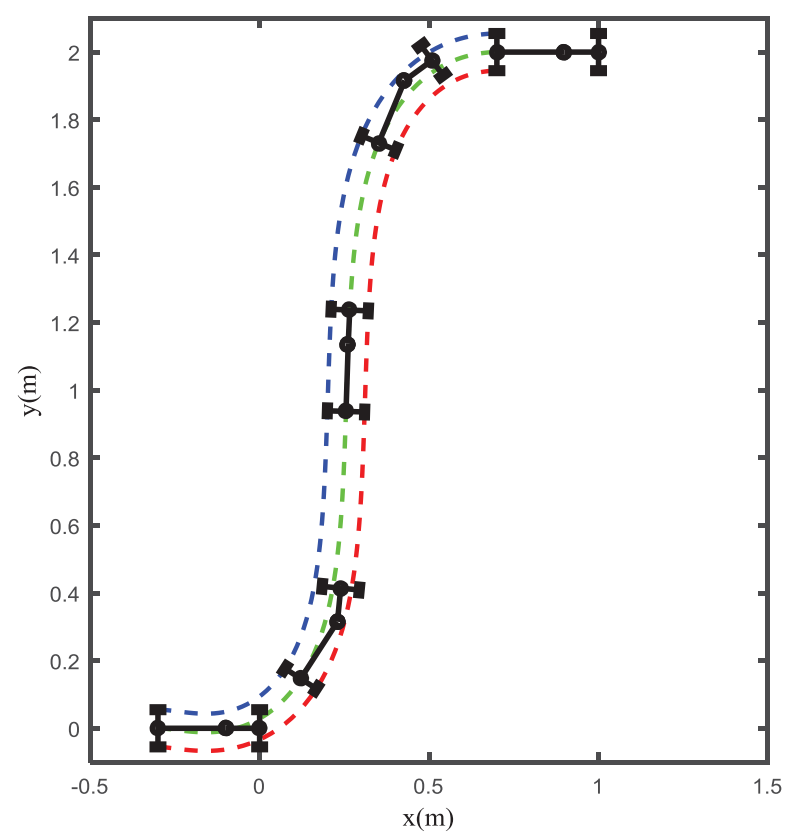

Figure 4 Optimal trajectory of FWTS

\section{Conclusions}

In this paper, the motion planning of FWTS is viewed as the optimal control problem subject to index-2 DAE with pure control constraints. To solve it, based on variation principle and nonsmooth theory, a novel symplectic numerical method is developed. Numerical simulation demonstrates that the proposed method is effective for motion planning of FWTS, the optimal motion planning is obtained and inequality constraints are satisfied.

\section{References}

[1] Y. Jing, Hierarchical motion planning for multisteering tractor-trailer mobile robots with on-axle hitching, IEEE-ASME Trans. Mechatron, 22(4):1652-1662, 2017.

[2] E. Kayacan, E. Kayacan, H. Ramon, and W. Saeys, Learning in centralized nonlinear model predictive control: application to an autonomous tractor-trailer system, IEEE Trans. Control Syst. Technol, 23(1):197-205, 2015.
[3] J. Chen and S. Kai, Cooperative transportation control of multiple mobile manipulators through distributed optimization, SCIENCE CHINA-INFORMATION SCIENCES, 61(12):120201, 2018.

[4] M. Yue, X. Hao,R. Gao, and J. Chen, Trajectory tracking control for tractor-trailer vehicles a coordinated control approach, Nonlinear Dynamics, 91:1061-1074, 2018.

[5] Y. Bian, M. Yang, X. Fang, and X. Wang, Kinematics and path following control of an articulated drum roller, Chinese Journal of Mechanical Engineering, 30(4):888-899, 2017.

[6] A. Khalaji and S. Moosavian, Robust adaptive controller for a tractor-trailer mobile robot, IEEE-ASME Trans. Mechatron, 19(3):943-953, 2014.

[7] M. A. S. de Aguiar, E. Camponogara, and B. Foss, An augmented Lagrangian method for optimal control of continuous time DAE systems, in IEEE Conference on Control Applications (CCA), Buenos Aires, Argentina, 2016.

[8] C. Jiang, K. Xie, C. Yu, M. Yu, H. Wang, Y. He, and K. L. Teo, A sequential computational approach to optimal control problems for differential-algebraic systems based on efficient implicit Runge-Kutta integration, Applied Mathematical Modelling, 58:313-330, 2018.

[9] C. Park, and D. J. Scheeres, Determination of optimal feedback terminal controllers for general boundary conditions using generating functions, Automatica, 42(5): 869-875, 2006.

[10] H. Peng, X. Wang, M. Li, and B. Chen, An hp symplectic pseudospectral method for nonlinear optimal control, Communications in Nonlinear Science and Numerical Simulation, 42:623-644, 2017.

[11] H. Peng, X. Wang, S. Zhang, and B. Chen, An iterative symplectic pseudospectral method to solve nonlinear state-delayed optimal control problems, Communications in Nonlinear Science and Numerical Simulation,48:95-114, 2017.

[12] H. R. Marzban, and S. M. Hoseini, A composite Chebyshev finite difference method for nonlinear optimal control problems, Communications in Nonlinear Science and Numerical Simulation, 18(6):1347-1361, 2013.

[13] M. Gerdts, Optimal Control of ODEs and DAEs, Berlin: Walter de Gruyter, 2012.

[14] M. Gerdts, and M. Kunkel, A globally convergent semi-smooth Newton method for control-state constrained DAE optimal control problems, Computational Optimization and Applications, 48(3):601-633, 2009.

[15] L. Qi, and J. Sun, A nonsmooth version of Newton's method, Mathematics Programming. 58:353-367,1993

[16] S. Ozana, M. Pies, and P. Wagner, Dynamic optimization of guide missile trajectory by use of MATLAB and DYNOPT toolbox, $12^{\text {th }}$ international conference on control, automation and systems(ICCAS), Jeju, South Korea, 2012. 